\title{
Intravenous immune globulin in hereditary inclusion body myopathy: a pilot study
} Susan Sparks ${ }^{1,2}$, Goran Rakocevic ${ }^{3}$, Galen Joe ${ }^{4}$, Irini Manoli ${ }^{1}$, Joseph Shrader ${ }^{4}$, Michael Harris-Love ${ }^{4,5}$, Barbara Sonies ${ }^{6}$, Carla Ciccone ${ }^{1}$, Heidi Dorward ${ }^{1}$, Donna Krasnewich ${ }^{1}$, Marjan Huizing ${ }^{1}$, Marinos C Dalakas ${ }^{3}$ and William A Gahl*1

\begin{abstract}
Address: ${ }^{1}$ Medical Genetics Branch, National Human Genome Research Institute, National Institutes of Health, Bethesda, MD, USA, ${ }^{2}$ Departmen of Genetics and Metabolism, Children's National Medical Center, Washington DC, USA, ${ }^{3}$ Neuromuscular Diseases Section, National Institute of Neurological Disorders and Stroke, National Institutes of Health, Bethesda, MD, USA, ${ }^{4}$ Department of Rehabilitation Medicine, Clinical Center, National Institutes of Health, Bethesda, MD, USA, ${ }^{5}$ George Washington University School of Medicine and Health Sciences, Washington DC, USA and ${ }^{6}$ University of Maryland, Department of Hearing and Speech Sciences, College Park, MD, USA

Email: Susan Sparks - ssparks@mail.nih.gov; Goran Rakocevic - rakocevg@ninds.nih.gov; Galen Joe - gjoe@cc.nih.gov; Irini Manoli - manolii@mail.nih.gov; Joseph Shrader - jshrader@cc.nih.gov; Michael Harris-Love - mhl@gwu.edu; Barbara Sonies - bsonies@comcast.net; Carla Ciccone - cciccone@mail.nih.gov; Heidi Dorward - hdorward@mail.nih.gov; Donna Krasnewich - dkras@mail.nih.gov; Marjan Huizing - mhuizing@mail.nih.gov; Marinos C Dalakas - dalakasm@ninds.nih.gov; William A Gahl* - bgahl@helix.nih.gov

* Corresponding author
\end{abstract}

Published: 29 January 2007

BMC Neurology 2007, 7:3 doi:10.1 186/147/-2377-7-3
Received: 12 September 2006

Accepted: 29 January 2007

This article is available from: http://www.biomedcentral.com/I47/-2377/7/3

(C) 2007 Sparks et al; licensee BioMed Central Ltd.

This is an Open Access article distributed under the terms of the Creative Commons Attribution License (http://creativecommons.org/licenses/by/2.0), which permits unrestricted use, distribution, and reproduction in any medium, provided the original work is properly cited.

\begin{abstract}
Background: Hereditary Inclusion Body Myopathy (HIBM) is an autosomal recessive, adult onset, non-inflammatory neuromuscular disorder with no effective treatment. The causative gene, GNE, codes for UDP-N-acetylglucosamine 2epimerase/ $\mathrm{N}$-acetylmannosamine kinase, which catalyzes the first two reactions in the synthesis of sialic acid. Reduced sialylation of muscle glycoproteins, such as $\alpha$-dystroglycan and neural cell adhesion molecule (NCAM), has been reported in HIBM.

Methods: We treated 4 HIBM patients with intravenous immune globulin (IVIG), in order to provide sialic acid, because lgG contains $8 \mu \mathrm{mol}$ of sialic acid/g. IVIG was infused as a loading dose of I g/kg on two consecutive days followed by 3 doses of 400 $\mathrm{mg} / \mathrm{kg}$ at weekly intervals.

Results: For all four patients, mean quadriceps strength improved from $19.0 \mathrm{~kg}$ at baseline to $23.2 \mathrm{~kg}(+22 \%)$ directly after IVIG loading to $25.6 \mathrm{~kg}(+35 \%)$ at the end of the study. Mean shoulder strength improved from $4.1 \mathrm{~kg}$ at baseline to $5.9 \mathrm{~kg}(+44 \%)$ directly after IVIG loading to $6.0 \mathrm{~kg}(+46 \%)$ at the end of the study. The composite improvement for 8 other muscle groups was $5 \%$ after the initial loading and $19 \%$ by the end of the study. Esophageal motility and lingual strength improved in the patients with abnormal barium swallows. Objective measures of functional improvement gave variable results, but the patients experienced improvements in daily activities that they considered clinically significant. Immunohistochemical staining and immunoblotting of muscle biopsies for $\alpha$-dystroglycan and NCAM did not provide consistent evidence for increased sialylation after IVIG treatment. Side effects were limited to transient headaches and vomiting.
\end{abstract}

Conclusion: The mild benefits in muscle strength experienced by HIBM patients after IVIG treatment may be related to the provision of sialic acid supplied by IVIG. Other sources of sialic acid are being explored as treatment options for HIBM. 


\section{Background}

Hereditary inclusion body myopathy (HIBM; OMIM 600737) is an adult-onset autosomal recessive myopathy that typically presents with distal muscle weakness, usually foot drop, in the second or third decade of life [1-3]. The disease progresses to require wheelchair confinement in two to three decades. The quadriceps muscles are relatively spared, at least clinically, even late in the disease. Muscle histology shows vacuolated fibers, variation of fiber size, increased connective tissue, and fibrofatty replacement of muscle fibers. Inflammation is typically absent.

HIBM is rare, but a Persian Jewish isolate [4] enabled investigators to map the condition to chromosome 9p1213. The causative gene, GNE, was subsequently identified and a common mutation, M712T, was found among affected Persian Jews [5]. The GNE gene encodes the bifunctional enzyme, UDP- $N$-acetylglucosamine (UDPGlcNAc) 2-epimerase/ $N$-acetylmannosamine (ManNAc) kinase, catalyzing the rate-limiting steps in sialic acid synthesis $[6,7]$. The M712T mutation is located within the kinase domain of the GNE gene. Nearly 20 other GNE mutations have been described in HIBM patients of different ethnic backgrounds [8-12]; a condition allelic to HIBM, Distal Myopathy with Rimmed Vacuoles (DMRV), occurs in Japan [13-15]. HIBM-associated GNE mutations, whether in the epimerase or the kinase domain, result in reduced activity of both UDP-GlcNAc 2-epimerase and ManNAc kinase $[16,17]$. These decrements are considered responsible for reduced production of sialic acid, a negatively charged sugar that serves as the terminal carbohydrate on glycoconjugates [18]. Glycosylation of proteins is critical for proper folding of nascent proteins, resistance to proteases, intracellular trafficking, and cellcell interactions $[19,20]$.

The pathogenic mechanism of muscle fiber degeneration in HIBM remains unknown. However, evidence suggests that decreased availability of sialic acid causes hyposialylation of muscle glycoproteins, whether involving glycans in general $[17,21]$, O-linked glycans [22], polysialic acid on neural cell adhesion molecules (PSA-NCAM) [23], or specific O-mannosylated glycosyl residues on $\alpha$ dystroglycan [24]. These latter oligosaccharides govern interactions of $\alpha$-dystroglycan with extracellular matrix proteins $[25,26]$, and their deficiency is responsible for several congenital muscular dystrophies, including Walker-Warburg syndrome, Muscle-Eye-Brain disease, and Fukuyama's muscular dystrophy [27-29]. In HIBM, decreased sialylation of $\alpha$-dystroglycan could impair interaction with other skeletal muscle proteins essential for function.
We reasoned that provision of sialic acid to HIBM patients could help normalize the sialylation status of muscle glycoproteins and provide clinical benefit. Because no approved source of free sialic acid is available, we delivered this charged sugar via intravenous immune globulin G (IVIG), a glycoprotein that contains $8 \mu$ moles of sialic acid per gram [30]. Maintaining high levels of serum IgG could result in continuous breakdown of the glycoprotein by neuraminidase, providing sufficient quantities of free sialic acid to the blood, scavenger cells and eventually muscle tissue. Side effects included headaches, nausea and vomiting, particularly after the second day of the loading dose; these resolved within 48 hours. Improvements in quantitative muscle testing and the six-minute walk were recorded. Qualitative improvements in activities of daily living, muscle strength, and endurance were noted in all four treated patients.

\section{Methods \\ Protocol design}

Four patients with molecularly proven HIBM were enrolled in a protocol approved by the NHGRI Institutional Review Board, and gave written, informed consent. Baseline evaluations at the NIH Clinical Center consisted of a complete history and physical examination, laboratory studies including quantitative immune globulins, a cardiac evaluation with a chest radiograph, electrocardiogram and echocardiogram, an ophthalmologic examination, and a neuromuscular evaluation that included an MRI of the calf and/or thigh. A baseline Rehabilitation Medicine evaluation included quantitative muscle strength testing, i.e., isometric fixed dynamometry [31] of 10 muscle groups, and measurements of tongue strength [32,33]. Strength was tested with subjects positioned supine and sitting on a treatment table, with stabilization provided by belts or examiners. Subjects performed two maximal voluntary isometric contractions of 10 muscle groups bilaterally separated by at least $30 \mathrm{~s}$ of rest. Joint angles were kept constant throughout the study via goniometric measurements. Cuffs were placed around the desired limb and were attached to a force transducer fixed to a stationary bed frame. The means of two trials were used for data analyses. Functional testing included a modified barium swallow evaluation, functional reach tests [34], timed up and go [34], and a 6-minute walk test to measure endurance [35]. A quadriceps muscle biopsy was taken for histology, immunohistochemistry and immunoblotting. Pulmonary function testing was performed in patients 3 and 4 .

After baseline testing, patients received a loading dose (1 $\mathrm{g} / \mathrm{kg}$ ) of immune globulin (Octagam 5\% or Gamunex $10 \%$ ) by continuous intravenous infusion on two consecutive days. Patients were hydrated before and after the infusions with $500 \mathrm{~mL}$ of either normal saline or half-nor- 
mal saline. They were also premedicated with $50 \mathrm{mg}$ of IV diphenhydramine and either $650 \mathrm{mg}$ of acetaminophen or $250 \mathrm{mg}$ of naproxen. One week after the two loading doses, the patients began receiving weekly maintenance doses of IVIG at $400 \mathrm{mg} / \mathrm{kg}$ for a total of 3 maintenance infusions. Hydration and premedication were also provided for these infusions. Evaluations of quantitative muscle testing, grip and pinch strength, 6-minute walk test and functional reach studies were performed directly after the loading dose and at the end of the study. Tongue strength measurements, a modified barium swallow, an ophthalmologic evaluation and a muscle biopsy were repeated at the end of the study. Laboratory testing was performed after each infusion and at the end of the study to monitor for safety and to follow levels of immune globulins.

\section{Muscle biopsies}

Open quadriceps muscle biopsies were performed before and 3-4 days after the last dose of IVIG. Specimens were processed for muscle enzyme histochemistry, immunocytochemistry, and immunoblotting as described below.

\section{Alpha-dystroglycan and NCAM immunocytochemistry} Coded serial cryosections (5 $\mu \mathrm{m}$ each), obtained before and after IVIG treatment from the patients, one normal control $\left(\mathrm{N}_{1}\right)$, and one individual with sporadic $\operatorname{IBM}\left(\mathrm{N}_{2}\right)$, were fixed in acetone for $5 \mathrm{~min}$ and pre-incubated with phosphate-buffered saline (PBS) containing $2 \%$ bovine serum albumin (BSA). The sections were then incubated overnight at $4{ }^{\circ} \mathrm{C}$ with antibodies against $\alpha$-dystroglycan (VIA4-1 and IIH6, Upstate, Lake Placid, NY, USA) diluted at 1:100, or anti-NCAM (123C3, Santa Cruz Biotechnologies, CA, USA) diluted at 1:200. Immunoreactivity was detected using FITC-labeled goat anti-mouse IgG or IgM (Molecular Probes, Eugene, OR) at a dilution of 1:500 for $1 \mathrm{~h}$. Negative controls included the omission of each primary antibody in every staining. The sections were mounted in $30 \%$ glycerol-based medium and viewed using fluorescent microscopy.

\section{Immunoblotting}

Muscle tissues were homogenized in $200 \mu \mathrm{L}$ of CelLytic (CelLytic, Sigma, St Louis, MO, USA), consisting of a mild detergent, bicine buffer and $150 \mathrm{mM} \mathrm{NaCl}$, with protease inhibitors (Complete Mini ${ }^{\circledast}$, Roche, Mannheim, Germany). The lysates were sonicated and microfuged at $8000 \mathrm{~g}$ for $10 \mathrm{~min}$ at $4^{\circ} \mathrm{C}$. The protein concentration of the supernatant was determined using the BCA protein assay (Pierce, Rockford, IL, USA) and equal amounts of protein $(25-50 \mu \mathrm{g})$ were loaded and electrophoresed on 4-12\% Tris-Glycine gels (Novex, Invitrogen, Carlsbad, CA, USA). Resolved proteins were electrophoretically transferred to a $0.45 \mu \mathrm{m}$ Hybond ECL nitrocellulose membrane (Amersham Pharmacia Biotech, IL, USA). Sub- sequently, membranes were blocked with $10 \%$ fat-free milk in PBS with $0.1 \%$ Tween 20 (PBST) for $30 \mathrm{~min}$ and then incubated with antibodies against NCAM (123C3, Santa Cruz Biotechnologies, CA, USA), $\alpha$-dystroglycan (clone IIH6C4, 05-593, Upstate, Lake Placid, NY, USA) or $\beta$-actin (AAN01, Cytoskeleton, Denver, CO, USA) followed by horseradish peroxidase-conjugated secondary antibodies in PBST containing 3\% fat-free milk. Immunoreactive bands were visualized by enhanced chemiluminescence using the Amersham ECL Western Blotting Detection Reagent (GE Healthcare, Buckinghamshire, UK). Density analysis was performed on digital images obtained with the Kodak Image station and software (Perkin Elmer, Boston, MA, USA). The protein levels were normalized to those of $\beta$-actin to correct for differences in protein loading and/or transfer.

For enzyme treatment experiments, protein samples (25 $\mu \mathrm{g}$ ) were incubated with neuraminidase (N-6514, Sigma, St Louis, MO, USA), $1 \mathrm{mU} / \mu \mathrm{g}$ of protein, for $30 \mathrm{~min}$ at $37^{\circ} \mathrm{C}$. Undigested and digested samples were analyzed by immunoblotting.

\section{Isoelectric focusing}

For isoelectric focusing (IEF) of transferrin, whole serum was iron-saturated and isofocused on pH4-6.5 PhastGels (Amersham Biosciences, Piscataway, NJ, USA) as described [36,37]. Transferrin isoforms were visualized by incubation with rabbit anti-human transferrin antibody (Dakocytomation, Denmark) followed by silver staining (Amersham Biosciences, Piscataway, NJ, USA). For IEF of apolipoprotein C-III (Apo C-III), whole serum proteins were acetone-delipidated and resuspended in $8 \mathrm{M}$ urea. Subsequently, Apo C-III was isolated and concentrated by centrifugation through centricon filters (Millipore, Bedford, MA, USA) and isofocused on PhastGels pH4-6.5 (Amersham Biosciences, Piscataway, NJ, USA). The proteins were transferred by diffusion blotting onto nitrocellulose membranes and visualized by incubation with goat anti-Apo CIII antibodies (Chemicon, Temecula, CA, USA) and Enhanced ChemiLuminescence detection (Amersham Biosciences, Piscataway, NJ, USA) as described $[37,38]$.

\section{Results \\ Patients}

Patient 1 is a 29 -year-old Iranian-Jewish woman diagnosed at age 24 years. She presented at age 17 with inability to abduct her legs against any resistance on an exercise machine; other muscle groups were strong. Following her first pregnancy at age 20, she noticed weakness, difficulty climbing stairs, and tripping and falling that progressed in frequency over the next 3 years from once a month to once a week. A muscle biopsy and genetic testing confirmed the diagnosis of HIBM with homozygous M712T 
mutations in GNE. At age 25, she noted difficulty fastening snaps and could not raise her arms to pull back her hair. Progression was gradual, with more significant decline following illnesses. Treatments included solumederol, $1 \mathrm{~g}$ intravenously for 3 days, which resulted in improved strength but was followed by relapse. On one occasion, IVIG (dose unknown) was given over 3 days, providing no improvement but complicated by aseptic meningitis requiring hospitalization. For the 6 months prior to admission, the patient used a wheelchair and required assistance walking and with transfers. Medications included sertraline for depression and anxiety, and vitamins.

At the NIH Clinical Center, the patient was wheelchair dependent, but could go from sit to stand and ambulate a few feet with support. She had bilateral foot drop and significant weakness of upper and lower extremities, with some sparing of the quadriceps. Muscle atrophy was universally apparent. Cranial nerves and sensation were intact. MRI of the calf showed pronounced fatty involution of the musculature bilaterally, worse proximally. The lateral soleus and posterior tibial muscles were somewhat spared distally. A quadriceps muscle biopsy revealed moderate type II fiber predominance. The muscles were atrophic (type II worse than type I) and demonstrated necrosis, active degeneration, and red rimmed vacuoles, consistent with severe chronic myopathy without inflammation. Imunohistochemical staining for NCAM, a surface glycoprotein reflecting muscle regeneration $[23,39]$, showed a significant number of positive fibers.

Patient 2 is the 42 -year-old brother of patient 1 . He was diagnosed with HIBM at 37 years of age, following the diagnosis of his sister. Weakness upon raising his arms overhead first appeared at age 27. At 34, he began tripping and, in the subsequent year, had difficulty climbing stairs and lifting his son. The upper extremities were most affected, but leg weakness was evident by decreased endurance, speed of walking, and getting in and out of a car. He occasionally used his hands to lift his legs. Symptoms were largely stable, but exacerbated by emotional events. He did not receive any treatment for his myopathy, and medications included only multivitamins.

At the NIH Clinical Center, there was wasting of the triceps and the interosseus and thenar muscles of the hand. Contractures of approximately 5-10 degrees were present in the elbows, with decreased range of motion upon supination of the forearm. The patient had no foot drop. Muscles of the shoulder girdle, pinch, grip, and proximal lower extremities exhibited weakness. The gait was normal, and cranial nerves and sensation were intact. MRI of the thigh revealed advanced atrophy with fatty involution in the proximal posterior compartment and adductor magnus muscles bilaterally. The sartorius and rectus femoris muscles were also involved. There was relative sparing of the quadriceps with the exception of the rectus femoris muscles. On biopsy, the quadriceps muscle showed several small vacuolated fibers with a few degenerating fibers, but no inflammation. Immunohistochemical staining was negative for NCAM, indicating negligible damage to individual muscle fibers.

Patient 3 is a 39-year-old woman of Bohemian/Czech/ French Canadian ancestry diagnosed with HIBM at the age of 31 years. Following a year of progressive tripping and falling, she awoke one day with numbness from the waist down that spread to her entire body. She was diagnosed with mild multiple sclerosis, based on the presence of oligoclonal bands in her cerebrospinal fluid and a brain MRI showing a possible "spot." Muscle biopsy also provided evidence of inclusion body myopathy. Currently, she has difficulty arising from a supine position due to weak abdominal muscles, weakness in her hands, arms, and shoulders, and inability to climb stairs. She wears ankle braces to walk. She uses bronchodilators for asthma and sertraline for depression. Confirmatory mutational analysis demonstrated compound heterozygous GNE mutations, i.e., R246Q and A631V, affecting the epimerase and kinase domains, respectively.

At the NIH Clinical Center, the patient had bilateral foot drop with a compensated gait. She was able to transfer independently from sit to stand and from stand to sit. There was proximal muscle and neck weakness and limited endurance. Cranial nerves and sensation were intact. MRI of the calf showed end-stage myopathy with advanced fatty involution, but with preservation of muscle volume. Signal alteration was present in areas of nonatrophic muscle. The popliteal muscles in the upper calves and the distal posterior tibial muscles were relatively spared. On biopsy, the quadriceps muscle showed significant necrosis with replacement of muscle by fat and connective tissue, but without inflammation. NCAM staining was minimal.

Patient 4 is the 46-year-old brother of patient 3. He was diagnosed with HIBM at the age of 33 years following a one-year history of progressive tripping and falling, decreased muscle strength and inability to lift his foot. As a cabinetmaker, he relied on this action to support the cabinets as he made them. A muscle biopsy was initially read as consistent with either polymyositis or inclusion body myopathy, but intravenous steroids provided no relief, and HIBM was diagnosed. Plateaus were interspersed with declines, which occurred with lack of exercise but not with illnesses. The lower extremity weakness extended throughout the body. The patient currently wears ankle braces to walk, uses an electric wheelchair for 
distances, and has assistive devices to drive. He has difficulty holding a pen. Medications include acyclovir, Metamucil, multivitamins, tramadol and diazepam.

At the NIH Clinical Center, there was muscle wasting of the triceps and the interosseus muscles of the hands, along with tightness of the heel cords, hips, and hamstrings. The patient was partially independent when going from sit to stand, and when executing transfers to and from his wheelchair. He could ambulate a few steps using ankle-foot orthotics and support from the wall. He had significant weakness in the proximal upper extremities, triceps, wrist and hand, and decreased strength on hip flexion, hip extension, ankle dorsiflexion, and plantar flexion. Deep tendon reflexes could not be elicited in the upper or lower extremities. Sensation and cranial nerve functions were intact. MRI of the calf showed end-stage myopathy with advanced fatty involution but preservation of muscle contours. Signal alteration occurred in areas of the remaining muscle. There was relative sparing of the popliteus, the tibialis posterior, and the flexor digitorum longus muscles. Quadriceps muscle histology revealed vacuolated and moth-eaten muscle fibers, with degeneration and regeneration. Connective tissue was slightly increased with no signs of inflammation. Immunohistochemical staining with NCAM was negative.

\section{Effects of IVIG on serum IgG}

IgG levels during the course of the study are shown in Figure 1 . The loading doses of IVIG brought the serum IgG levels to approximately 4 times the baseline, but this concentration of IgG was not sustained by the weekly infusions of $400 \mathrm{mg} / \mathrm{kg}$ of IVIG. Throughout the course of the infusion, the patients' serum IgG concentrations ranged from 2 to 4 times the average normal level.

\section{Muscle strength}

On quantitative muscle testing, both distal and proximal muscle groups showed improvement after IVIG treatment. The greatest absolute improvement was in quadriceps strength, as reflected in the capacity to perform work in knee extension. Considering both sides of all 4 patients, mean quadriceps strength improved from $19.0 \mathrm{~kg}$ at baseline, to $23.2 \mathrm{~kg}(+22 \%)$ directly after IVIG loading, to 25.6 $\mathrm{kg}(+35 \%)$ at the end of the study. Considered individually, the 4 patients exhibited a $13-150 \%$ improvement in the dominant right leg after IVIG treatment (Figure 2). The change in left leg quadriceps strength varied from $-3 \%$ to $+48 \%$. Shoulder abduction also showed significant improvement. Considering both sides of all 4 patients, mean shoulder strength improved from $4.1 \mathrm{~kg}$ at baseline, to $5.9 \mathrm{~kg}(+44 \%)$ directly after IVIG loading, to $6.0 \mathrm{~kg}$ $(+46 \%)$ at the end of the study. Considering each patient individually, shoulder abduction on the right side improved $24-79 \%$ in three patients and fell $14 \%$ in patient 3 (Figure 2). On the left side, shoulder abduction improved 13-184\%.

Eight other muscle pairs (right and left), associated with hip flexion, ankle dorsiflexion, elbow flexion and extension, wrist flexion and extension, grip, and pinch, were also evaluated. These muscle groups exhibited variable differences in strength before and after IVIG treatment (Table 2), but the preponderance showed improvement. In fact, on average, the composite improvement for these 8 muscle groups was 5\% after the initial IVIG loading and $19 \%$ by the end of the study. Tongue strength, which is not known to be affected in HIBM, improved 5\%, from a mean of $63.1 \mathrm{kp}$ at baseline to a mean of $66.2 \mathrm{kp}$ at the end of the study.

\section{Functional testing}

Functional studies included functional reach, timed up and go, and the 6-min walk test. Patient 1 could not perform any of these tests. Patient 4 could not perform the 6min walk test.

In patient 2, the functional reach after IVIG treatment increased from 7.3 in to 10.2 in (40\%) on the right and from 6.0 in to 8.8 in (47\%) on the left. However, the functional reach declined in patients 3 and 4 by $8 \%$ and $19 \%$, respectively. The time it took to rise from a chair (timed up and go) improved $1.94 \mathrm{~s}$ (27\%) for patient 2 and 0.81 $\mathrm{s}(8 \%)$ for patient 3 ; it worsened by $0.84 \mathrm{~s}(5 \%)$ in patient 4. Endurance, as measured by the 6-min walk test, improved after IVIG treatment from $1633 \mathrm{ft}$ to $1744 \mathrm{ft}$ $(7 \%)$ in patient 2 (normal mean for age and size, $1997 \mathrm{ft}$ ) and from $983 \mathrm{ft}$ to $1065 \mathrm{ft}(8 \%)$ in patient 3 (normal mean, $1637 \mathrm{ft}$ ).

Improvements in the two patients with abnormal modified barium swallows were also noted. Patient 1 had normal esophageal peristalsis with only mild delays after IVIG and patient 3 had no reflux or pooling with only delayed initiation after the treatment.

Patients displayed qualitative or subjective improvements as well. Patient 1 claimed improved strength, energy, and balance and needed less support to walk. She could get in and out of a bed or chair more easily. Brushing her hair was easier. She was able to open a bottle of water and cut a bagel without help. After IVIG treatment, she could snap her fingers and produce noise, which she had been unable to do for years. Patient 2 noted improvements in getting out of bed or a car. He could easily wash his hands and face, which was a struggle before treatment. He could reach further and could lift his children; he had more energy to play with them. Both he and his wife noticed that he walked faster. Patient 3 noted improvements with getting out of bed and up from a sitting position. She 


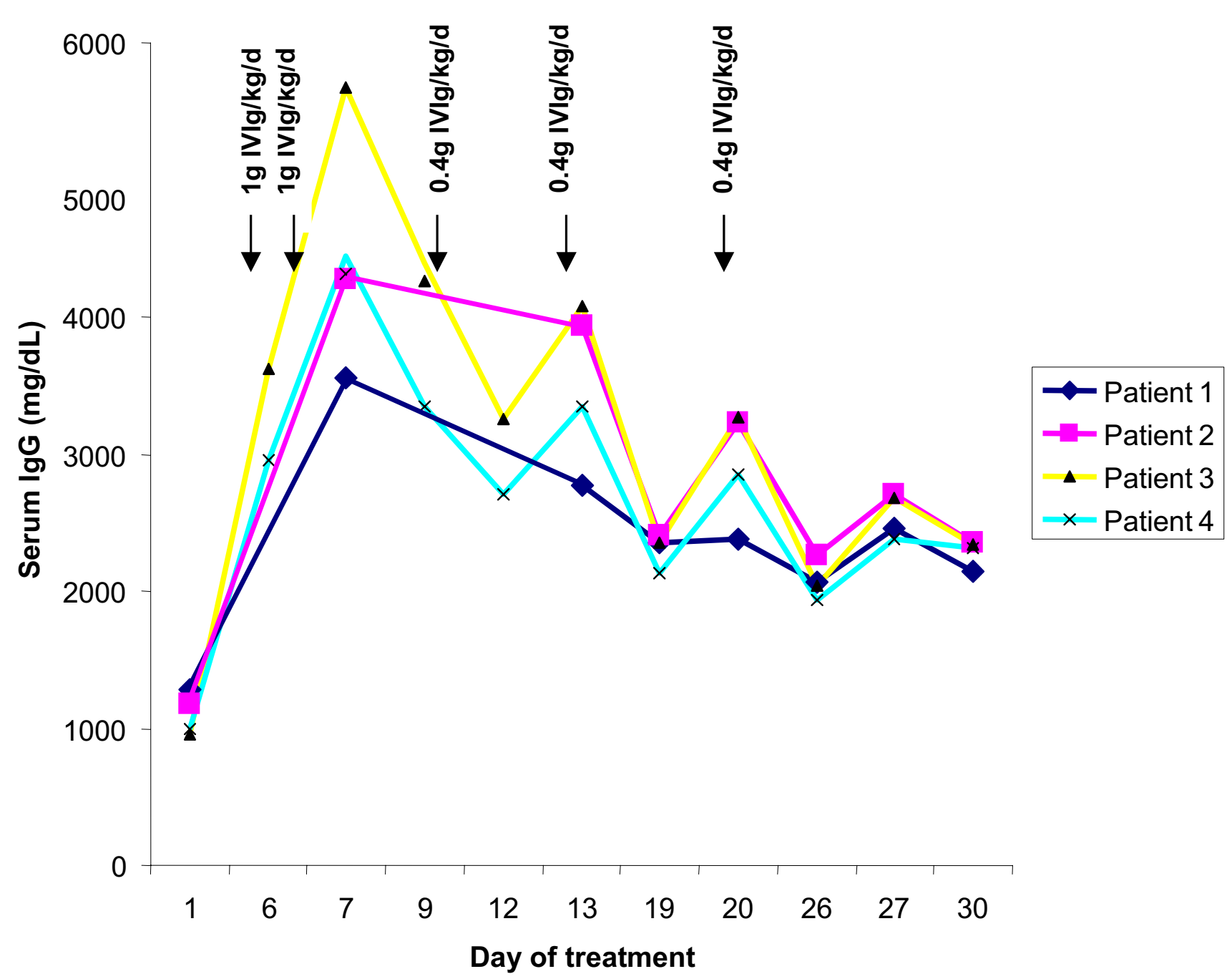

Figure I

Serum IgG levels in patients during the course of treatment with IVIG. Arrows indicate loading doses (I g/kg) and maintenance doses $(0.4 \mathrm{~g} / \mathrm{kg})$.

needed less support to accomplish her activities of daily living and appreciated increased energy. Patient 4 stated that he felt better, but was unable to provide specific examples. The subjective improvements lasted approximately three weeks, after which each of the patients noted a decline in function.

\section{Serum glycoproteins}

Serum transferrin contains four terminal sialic acid residues on its N-linked oligosaccharides [36,37]. On isoelectric focusing, all four oligosaccharides were sialylated in all four patients before IVIG treatment, just after loading, and at the end of the treatment period [see Additional file 1]. Similarly, the O-linked serum glycoprotein Apo C-III $[37,38]$ exhibited a normal contingent of sialylation in all four patients before and after IVIG treatment [see Additional file 1].

\section{Muscle studies}

Muscle MRI examinations were not repeated after IVIG treatment, because one month was not deemed a sufficient amount of time to achieve visible improvement. Standard histology of muscle biopsies showed no difference before compared with after IVIG treatment. The posttreatment biopsy of patient \#3 was unsuitable for interpretation, but muscle biopsies from patients 1,2 , and 4 before and after IVIG treatment were available for staining with anti- $\alpha$-dystroglycan antibodies (VIA4I and IIH6). No consistent differences were seen before and after treatment (Figure 3A-F). Immunoblotting showed no consistent increase in the glycosylation status of $\alpha$-dystroglycan 


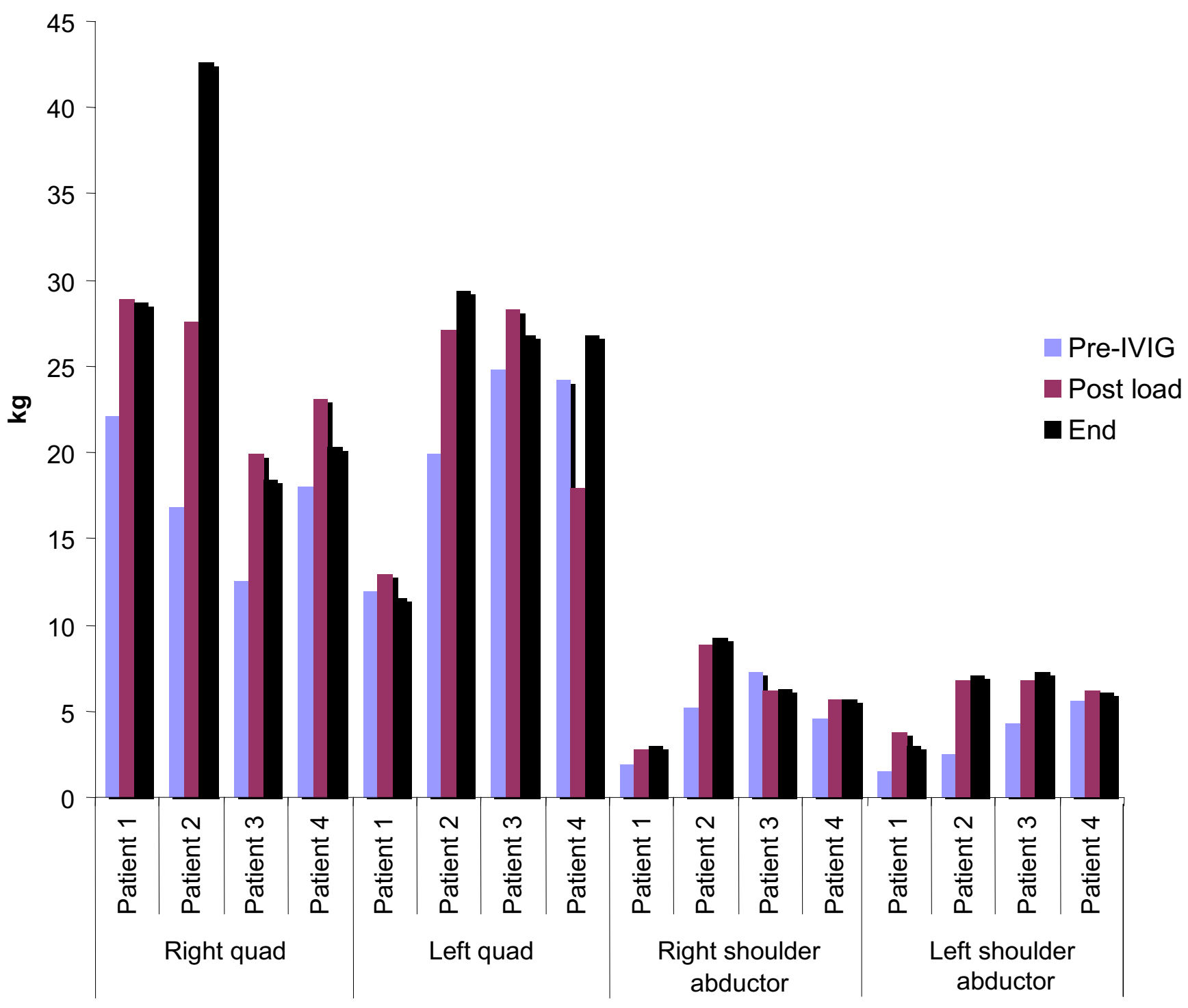

Figure 2

Quadriceps and shoulder abduction testing in four patients with HIBM. On average, quadriceps strength increased $22 \%$ after IVIG loading and $35 \%$ at the end of the study. Shoulder abduction strength increased $44 \%$ after IVIG loading and $46 \%$ at the end of the study.

(using the IIH6 antibody) after IVIG treatment (Figure $3 G)$.

NCAM is a sialylated glycoprotein that plays a significant role in muscle fiber development, innervation and regeneration, and whose abundance on the surface of muscle cells reflects the extent of muscle denervation or damage [23,39-42]. Patient 1 exhibited the greatest amount of muscle NCAM before IVIG (Figure 4A), i.e., 4 -fold that of the $\mathrm{N}_{1}$ (normal) control, relative to actin, as determined by densitometry. After IVIG treatment, the muscle NCAM of patient 1 decreased by $34 \%$. The amount of NCAM decreased by $28 \%$ in patient 2 , but increased by $58 \%$ in patient 4 , relative to actin after IVIG administration. In all cases, the HIBM patients' NCAM bands ran lower than the control, reflecting a decreased sialic acid content of this glycoprotein. This was verified for patient 2 by using sialidase to remove terminal sialic acid residues (Figure 4B). Sialidase treatment resulted in greater mobility of the normal muscle NCAM protein, but not that of patient 2 (Fig- 
Table I: Laboratory values at baseline, after loading with IVIG (I g/kg $\times 2)$, and at the end of IVIG treatment.

\begin{tabular}{|c|c|c|c|c|c|c|c|c|c|c|c|c|c|}
\hline \multirow[b]{2}{*}{ Patient } & \multicolumn{4}{|c|}{ Baseline } & \multicolumn{4}{|c|}{ Post-load } & \multicolumn{4}{|c|}{ End of study } & \multirow[t]{2}{*}{ Normal range } \\
\hline & I & 2 & 3 & 4 & I & 2 & 3 & 4 & I & 2 & 3 & 4 & \\
\hline Hemoglobin (g/dL) & 13.1 & 14.4 & 13.2 & 14.3 & 11.2 & 13.8 & 11.2 & 13 & 12.1 & 13.8 & 12.3 & II.4 & $11.1-16.7$ \\
\hline WBC (K/uL) & 8.94 & 7.57 & 7.39 & 7.28 & 6.82 & 6.11 & 8.06 & 2.99 & 6.94 & 6.16 & 6.34 & 4.85 & $3.3-9.6$ \\
\hline Platelets (K/uL) & 282 & 230 & 284 & 225 & 226 & 209 & 262 & 207 & 267 & 222 & 256 & 220 & $154-380$ \\
\hline ESR (mm/h) & 17 & 7 & 23 & 14 & 39 & 38 & 96 & 86 & 30 & 20 & 57 & 73 & $0-42$ \\
\hline Creatinine (mg/dL) & 0.3 & 0.7 & 0.5 & 0.4 & 0.4 & 0.8 & 0.6 & 0.6 & 0.3 & 0.7 & 0.6 & 0.5 & $0.7-1.4$ \\
\hline BUN (mg/dL) & 15 & 14 & 12 & 13 & 12 & 7 & 8 & 8 & 16 & 15 & 13 & 17 & $8-22$ \\
\hline ALT (U/L) & 42 & 36 & 18 & 36 & 31 & 37 & 16 & 30 & 60 & 81 & 18 & 62 & $6-41$ \\
\hline AST (U/L) & 30 & 31 & 22 & 23 & 21 & 31 & 15 & 23 & 36 & 68 & 31 & 52 & $9-34$ \\
\hline CPK (U/L) & 320 & 475 & 98 & 130 & 244 & 451 & 50 & 87 & 229 & 629 & 78 & 94 & $38-386$ \\
\hline Cholesterol (mg/dL) & 195 & 203 & 195 & 207 & 133 & 153 & 144 & 156 & 168 & 179 & 175 & 170 & $100-200$ \\
\hline Total protein (g/dL) & 6.4 & 6.1 & 6.1 & 6.3 & 7.8 & 8.7 & 9.5 & 9.1 & 7.1 & 7.2 & 7.3 & 7 & $6.0-7.6$ \\
\hline Albumin (g/dL) & 3.7 & 3.7 & 3.2 & 3.5 & 2.9 & 3.4 & 2.6 & 3 & 3.3 & 3.5 & 2.8 & 3.1 & $3.7-4.7$ \\
\hline IgG (mg/dL) & 1280 & 1180 & 955 & 997 & 3550 & 4280 & 5670 & 4440 & 2150 & 2350 & 2340 & 2310 & $642-1730$ \\
\hline Urine protein $(\mathrm{mg} / \mathrm{d})$ & 150 & 92 & 294 & 97 & $<30$ & $<30$ & $<30$ & $<30$ & 79 & 95 & 364 & 142 & $30-150$ \\
\hline Urine glucose (g/d) & 0.07 & 0.07 & 0.07 & 0.09 & 14.4 & 30.8 & 6.4 & 12.2 & 0.07 & 0.07 & 0.10 & 0.08 & $0-0.49$ \\
\hline
\end{tabular}

Abnormal values are in bold. Normal range encompasses values for males and females.

Baseline values are average of results on two different days.

ure $4 \mathrm{~B})$. Sialidase treatment did, however, reduce the apparent size of muscle NCAM in patient 1 , indicating the presence of some sialylation.

NCAM immunohistochemical staining of HIBM muscle (patient 1, 2, and 4) after IVIG treatment did not reveal any consistent differences compared with pre-treatment staining (data not shown).

\section{Side effects}

All patients experienced fatigue and headache following the second loading dose of $1 \mathrm{~g} / \mathrm{kg}$ IVIG. Patients 1,2 , and 3 had several episodes of nausea and vomiting; patient 2 said it resembled having influenza. Patient 4 had headache and neck stiffness. Patients 1 and 2 experienced anorexia, and patient 2 had abdominal distension and constipation. The headaches were treated with naproxen or acetaminophen. Patient 3 required zofran for relief of and did not recur with subsequent maintenance infusions of IVIG. A urinary tract infection was diagnosed incidentally in patient 1 , and was treated. The follow-up ophthalmologic evaluation in patient 4 revealed a cottonwool spot near the left optic disc.

Patients 3 and 4 responded to the IVIG with increased sedimentation rates, as high as $96 \mathrm{~mm} / \mathrm{h}$ (Table 1). Liver enzymes rose slightly in patients 1, 2 and 4; the highest levels were obtained in patient 2, with an ALT of $81 \mathrm{U} / \mathrm{L}$ and an AST of $68 \mathrm{U} / \mathrm{L}$. These values subsequently returned to normal. Serum cholesterol levels generally fell, from a mean of $200 \mathrm{mg} / \mathrm{dL}$ at baseline to $147 \mathrm{mg} / \mathrm{dL}$ just after loading and $173 \mathrm{mg} / \mathrm{dL}$ at the end of the study. Remarkably, the four patients excreted 6.4 to $30.8 \mathrm{~g} / \mathrm{d}$ of glucose in their urine just after the IVIG load, compared with $\leq 0.1 \mathrm{~g} /$ day before and after the study. There were no significant changes in serum electrolytes, glucose, calcium, phospho-

Table 2: Strength of muscle groups of 4 HIBM patients at baseline and after IVIG treatment.

\begin{tabular}{|c|c|c|c|c|c|c|c|c|}
\hline & \multirow[t]{2}{*}{ Hip Flexion } & \multirow[t]{2}{*}{ Ankle Dorsiflexion } & \multicolumn{2}{|c|}{ Elbow } & \multicolumn{2}{|c|}{ Wrist } & \multicolumn{2}{|c|}{ Hand } \\
\hline & & & Flexion & Extension & Flexion & Extension & Grip & Pinch \\
\hline Baseline $^{a, b}$ & $3.21 \pm 0.84$ & $3.50 \pm 2.15$ & $4.71 \pm 1.42$ & $3.36 \pm 1.09$ & $5.24 \pm 0.86$ & $4.55 \pm 0.46$ & $7.43 \pm 1.63$ & $1.02 \pm 0.22$ \\
\hline Post-Loadb & $\begin{array}{c}3.93 \pm 0.74 \\
(+22 \%)\end{array}$ & $\begin{array}{c}2.53 \pm 1.55 \\
(-28 \%)\end{array}$ & $\begin{array}{c}5.56 \pm 1.60 \\
(+18 \%)\end{array}$ & $\begin{array}{c}3.30+0.98 \\
(-2 \%)\end{array}$ & $\begin{array}{c}5.81 \pm 1.07 \\
(+11 \%)\end{array}$ & $\begin{array}{c}5.15 \pm 0.58 \\
(+13 \%)\end{array}$ & $\begin{array}{c}7.85 \pm 1.65 \\
(+6 \%)\end{array}$ & $\begin{array}{c}1.03 \pm 0.16 \\
(+1 \%)\end{array}$ \\
\hline End of studyb & $\begin{array}{c}3.63 \pm 0.62 \\
(+13 \%)\end{array}$ & $\begin{array}{l}4.28 \pm 2.87 \\
(+22 \%)\end{array}$ & $\begin{array}{c}5.45 \pm 1.55 \\
(+16 \%)\end{array}$ & $\begin{array}{c}3.36 \pm 1.03 \\
(0 \%)\end{array}$ & $\begin{array}{c}6.25 \pm 1.15 \\
(+19 \%)\end{array}$ & $\begin{array}{c}5.78 \pm 0.93 \\
(+27 \%)\end{array}$ & $\begin{array}{c}7.83 \pm 1.59 \\
(+5 \%)\end{array}$ & $\begin{array}{c}1.37 \pm 0.25 \\
(+35 \%)\end{array}$ \\
\hline
\end{tabular}

a Values are given in $\mathrm{kg}$ as means \pm SEM. Percent changes are in parentheses. Values for both the right and left sides were included in the analyses. bBaseline: Before treatment; Post-Load: Day I after IVIG load (I g/kg × 2); End of study: 4 weeks after the initial IVIG load.

the nausea and vomiting. All symptoms resolved by $72 \mathrm{~h} \quad$ rus, or bilirubin. 

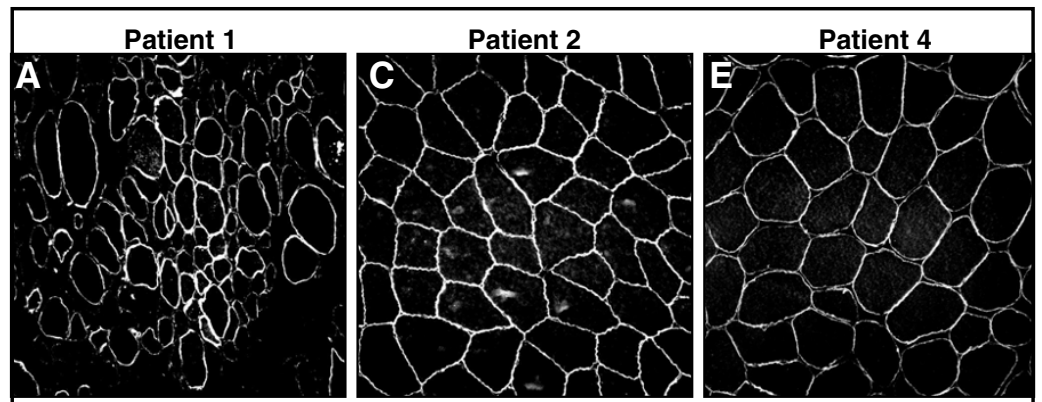

Pre IVIG
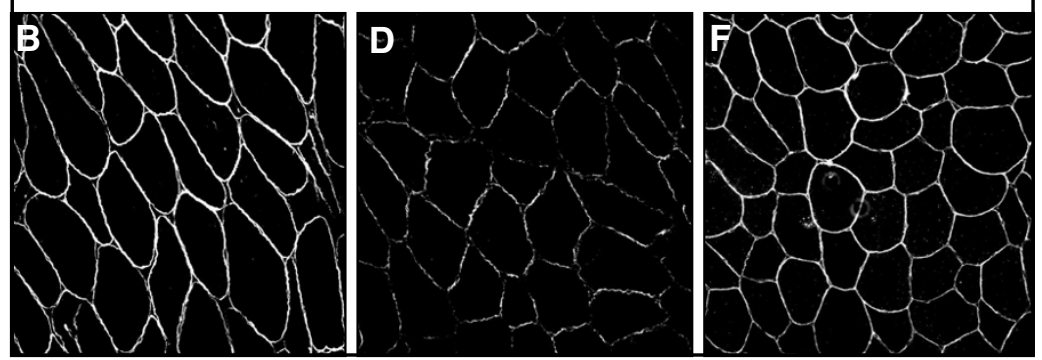

G

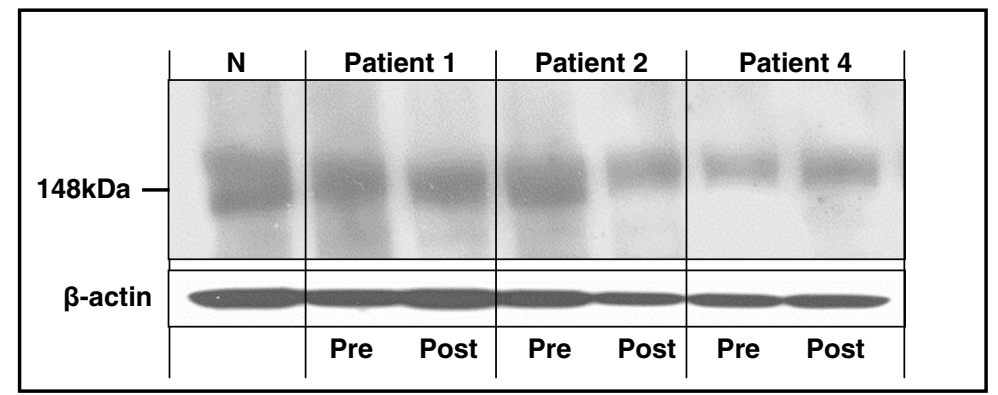

Figure 3

Expression of $\alpha$-dystroglycan in quadriceps muscle from HIBM patients. A-F. Immunohistochemistry using antibodies to IIH6. No consistent difference in staining was apparent before (A,C,E) compared with after (B,D,F) IVIG treatment. The specimen shown in $A$ was sampled from a deteriorating area. $\mathbf{G}$. Immunoblots of muscle from a normal individual (N) and HIBM patients I, 2, and 4, labeled with antibodies to $\alpha$-dystroglycan. No consistent difference in IIH6 staining (reflecting the sialylation status of $\alpha$-dystroglycan) was apparent before (Pre) compared with after (Post) IVIG treatment. $\beta$-Actin bands provide an indication of the level of protein loading.

\section{Discussion}

Homozygous or compound heterozygous mutations in either the epimerase or kinase domain of GNE cause decreased UDP-GlcNAc 2-epimerase and ManNAc kinase activities in the cells of HIBM patients [16,17]. However, the pathogenic mechanisms responsible for the myopathy of HIBM have not been determined. Measurements of the sialic acid content of glycoproteins have given variable results $[17,21-24,43-45]$. Using sialylated $\alpha$-dystroglycanspecific antibodies, Huizing et al. first reported decreased muscle $\alpha$-dystroglycan sialylation in four HIBM patients, each having one mutation in the epimerase domain and one in the kinase domain of GNE [24]. Noguchi et al. followed with findings of fiber-to-fiber variability in the hyposialylation of $\alpha$-dystroglycan in the muscles of patients with DMRV, allelic with HIBM [17]. Saito et al. reported hyposialylation of muscle glycoproteins in a single patient with DMRV [21], but Hinderlich et al. and Salama et al. showed no decrease in sialylation of proteins in myoblasts cultured from HIBM patients homozygous for the Persian Jewish GNE mutation, i.e., M712T [43,44]. Broccolini et al. recently reported hyposialylated muscle $\alpha$-dystroglycan in 4 of 5 HIBM patients, with no effect on binding to laminin [45]; they surmised that the sialylation status of $\alpha$-dystroglycan is not critical to the protein's function. Ricci et al. used NCAM as an indicator of the extent of muscle glycoprotein sialylation; in HIBM, muscle NCAM was found to be hyposialylated [23]. The divergence of these findings allows for the possibility that sialylation abnormalities represent epiphenomena in HIBM, and GNE mutations could cause disease by affecting cellular functions not influenced by sialylation. 


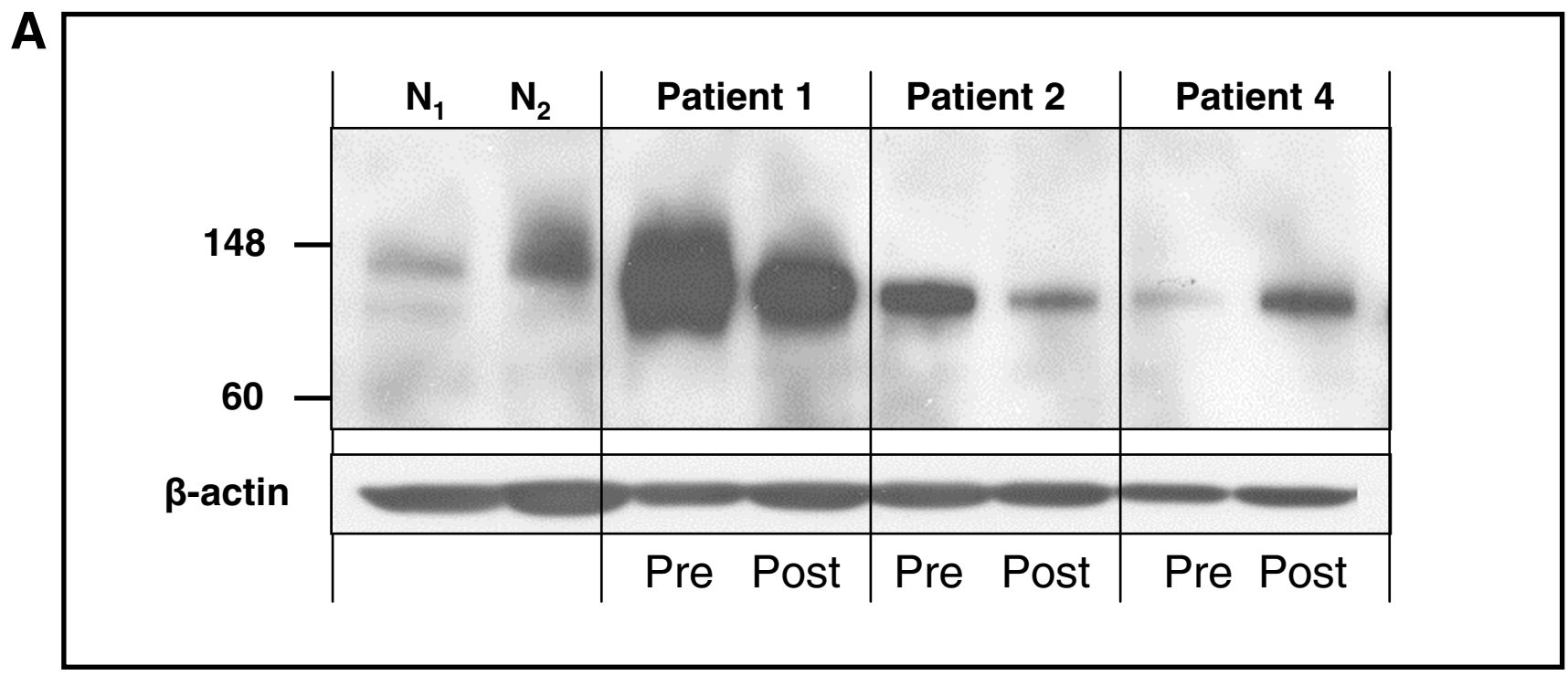

B

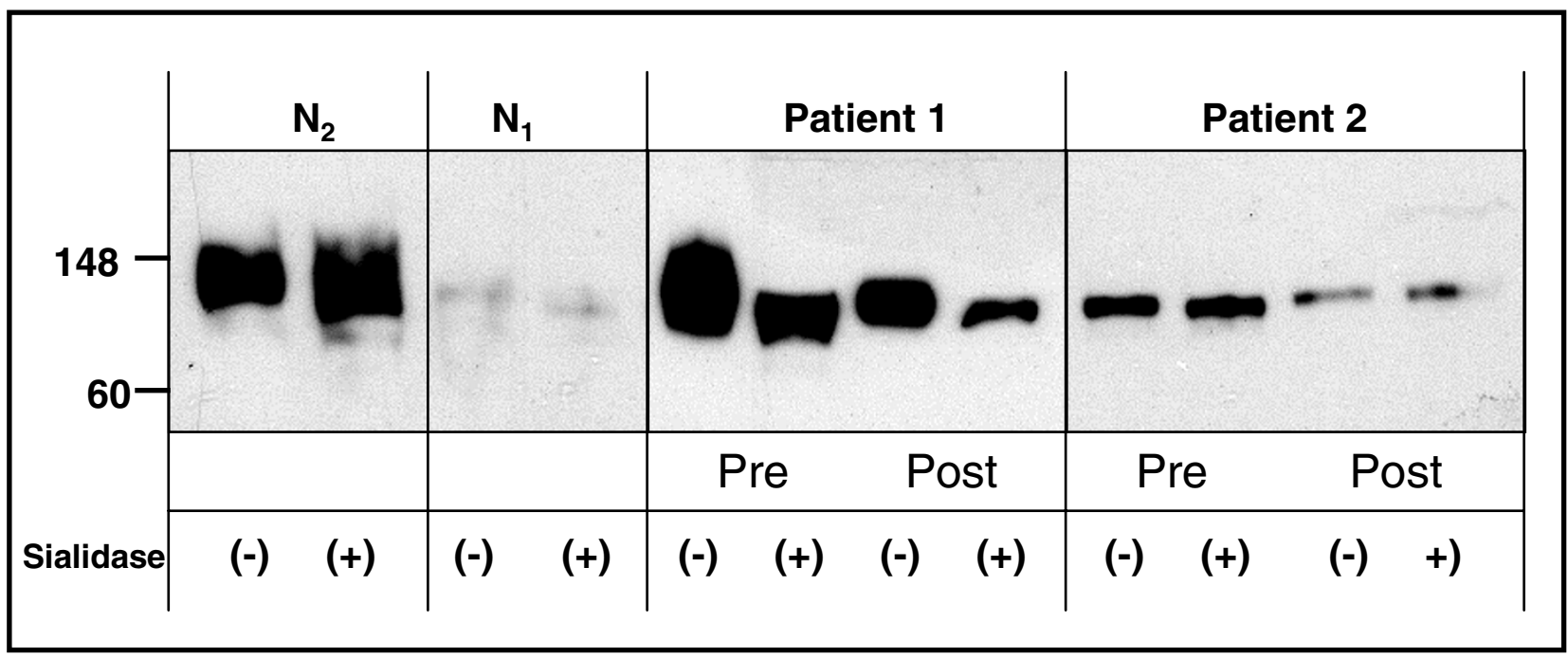

\section{Figure 4}

Expression of sialylated NCAM in muscle of HIBM patients. A. Muscle from two control individuals exhibit NCAM mobility consistent with a higher molecular weight, indicative of sialylation. $N_{1}$ is normal and $N_{2}$ has sporadic IBM; note increased NCAM in this person's muscle. The muscle of patient I also has an increased amount of NCAM; patients 2 and 4 have moderate amounts of NCAM. The NCAM of the HIBM patients migrates with greater mobility than normal, indicating lack of sialylation. The amount of muscle NCAM decreased after (Post) compared with before (Pre) IVIG treatment in patients I and 2, but increased in patient 4. B. Sialidase treatment increased the mobility of control muscle $\left(N_{1}\right.$ and $\left.N_{2}\right)$. The mobility of muscle NCAM was not altered by sialidase treatment in patient 2, but it was increased by sialidase treatment in patient $\mathrm{I}$.

Indeed, while we verified the hyposialylation of muscle NCAM in our HIBM patients (Figure 4A,B), we found that decreased sialylation of glycoproteins is not a constant finding in HIBM. Isoelectric focusing studies revealed normal N-linked glycosylation of serum transferrin and $\mathrm{O}-$ linked glycosylation of Apo C-III [see Addional file 1]. In addition, sialylated $\alpha$-dystroglycan was not decreased in the muscle of our four HIBM patients prior to IVIG administration. This could be because patients vary with respect to their specific GNE mutations, the extent of their sialic acid deficiency, and the degree to which their muscle proteins are sialylated, even when sampling the same 
muscle. Conditions of the immunohistochemical staining could differ, as could the specificity and sensitivity of the antibodies.

With normal baseline glycosylation of serum glycoproteins and muscle $\alpha$-dystroglycan in HIBM, we had no opportunity to demonstrate improvement in these parameters after IVIG treatment. Muscle NCAM was hyposialylated before IVIG administration, but we could not discern consistent improvements in NCAM sialylation, either by immunohistochemistry or by immunoblotting, after IVIG treatment. One possible explanation is that the amount of sialic acid provided by IVIG was inadequate to increase glycoprotein sialylation. We estimate that normal adult urinary free sialic acid, a reasonable measure of daily production, approximates $0.3 \mathrm{mmol}$ [46]. Our IVIG loading doses $(2 \mathrm{~g} / \mathrm{kg})$ should provide $8 \mu \mathrm{mol} / \mathrm{g}$ or $\sim 1 \mathrm{mmol}$ of sialic acid, roughly 3 days' worth of normal sialic acid production. This may or may not be sufficient to achieve a detectable or functional increase in the glycosylation of muscle protein.

We would expect a decrease in muscle NCAM to accompany the decrease in muscle degeneration hoped for with IVIG treatment, and this occurred in patients 1 and 2. The increased amount of hyposialylated NCAM in patient 4 after IVIG treatment contradicts this finding and reflects variability either in response to treatment or in muscle sampling.

In contrast to the absence of consistent effects on histological and immunological markers of sialylation, IVIG treatment improved objective and subjective measures of muscle strength and function in HIBM patients. Muscles that had greater strength at baseline, such as the quadriceps, appeared to benefit more than muscles that were already extensively damaged; results were variable but, on average, the improvement in strength exceeded 20\% in the quadriceps and shoulders. Even though the acquisition of functional abilities was short-lived, all the patients were pleased, and two of them wished to pursue IVIG treatments independently. It is not surprising that acute clinical improvements were not accompanied by histological changes, since morphological changes should require long-term treatments and, perhaps, muscle regeneration.

There are several possible explanations for the beneficial clinical effects of IVIG despite the absence of evidence for increased glycoprotein sialylation. The salutary responses to IVIG could have been due to its anti-inflammatory actions $[47,48]$; patient 3 had previous evidence of central nervous system inflammation and patient 4 had a muscle biopsy read as possible polymyositis. Nevertheless, the myopathy in HIBM is not considered to be inflammatory, and muscle biopsies performed at the NIH on all four patients lacked signs of inflammation. A second possibility is that minor increases in sialylated muscle glycoproteins, responsible for improved muscle function, were present transiently after IVIG treatment, but were undetectable in our assay. A third possibility is that the muscle we biopsied, the quadriceps, had minimal baseline reduction in glycoprotein sialylation so that any increased sialylation after therapy could not be appreciated. Finally, the beneficial clinical effects might not be at all related to sialylation.

Ours was clearly a pilot study, clarifying some issues for future therapeutic investigations. For example, we documented that muscle strength testing provides an objective outcome parameter, with fixed dynamometry detecting small and large changes in strength, complemented by more subjective measures of muscle function [49]. We also determined that muscle histology, immunohistology and sialylated glycoprotein quantitation by immunoblotting do not provide reliable measures of improvement, at least in the short term. These parameters require further investigation using longer treatment regimens and greater amounts of sialic acid supplementation. One possibility would be to administer only the Fc portion of immune globulin, which contains the bulk of the sialic acid residues and has ten times the anti-inflammatory efficacy of the complete molecule [47]. Even so, IVIG is not likely to be the treatment of choice for HIBM, because it must be administered repeatedly, its mode of delivery requires hospitalization, its cost is considerable, and it has side effects. However, the finding of definitive improvements, albeit moderate and transient, attributable to IVIG suggests that the provision of sialic acid holds therapeutic promise.

Our next pursuit will involve administration of ManNAc, an uncharged sugar situated in the sialic acid synthetic pathway after the rate-limiting UDP-GlcNAc 2-epimerase step. Residual ManNAc kinase activity in HIBM patients, or ancillary kinases such as GlcNAc kinase [50], might convert ManNAc into ManNAc-6P for subsequent synthesis of sialic acid. In fact, hyposialylated, GNE-deficient mouse embryonic stem cells became resialylated after their growth medium was supplemented with ManNAc [51]. Furthermore, ManNAc supplementation has resulted in prolonged survival of a mouse model of HIBM [52], and could yield increased glycoprotein sialylation in the tissues of humans with HIBM.

\section{Conclusion}

Our findings indicate that IVIG treatment has a salutary clinical effect on the myopathy of HIBM. Since HIBM results from defective sialic acid biosynthesis and is not associated with inflammation, the benefits of IVIG treatment could be related to the high sialic acid content of 
IgG. These results prompt consideration of other sources of sialic acid as therapy for HIBM, and identify suitable outcome parameters for future clinical trials.

\section{Abbreviations}

HIBM, Hereditary Inclusion Body Myopathy; UDP-GlcNAc, uridine diphosphate-N-acetylglucosamine; ManNAc, N-acetylmannosamine; IVIG, intravenous immune globulin G, NCAM, neural cell adhesion molecule; IEF, isoelectric focusing

\section{Competing interests}

The author(s) declare that they have no competing interests.

\section{Authors' contributions}

SS contributed to conception and design, clinical organization and data collection, and drafting of the manuscript; GR collected the muscle samples and performed immunohistochemistry; MH-L designed the quantitative muscle strength protocol; GJ and JS performed quantitative muscle strength and functional testing; IM and CC performed biochemical analyses including isoelectric focusing and immunoblotting; BS performed swallowing evaluations and tongue strength measurements; HD provided imaging support for immunohistochemistry and preparation of figures; DK contributed to conception and design; $\mathrm{MH}$ contributed to conception and design, supervised laboratory analysis and interpretations, and drafting the manuscript; MCD contributed to conception and design and provided clinical expertise on IVIG treatment and muscle histology; WAG substantially contributed to trial conception and design, drafting of the clinical protocol for IRB approval, and drafting the manuscript. All authors read and approved the final manuscript.

\section{Additional material}

\section{Additional file 1}

Isoelectric focusing of serum transferrin and Apo C-III. A. Normal sialylation of transferrin (N-linked glycoprotein) in all four patients before IVIG (Pre), after IVIG loading (Mid), and after the treatment period (Post). Samples of patients 3 and 4 were electrophoresed on a gel different from the gel for samples 1 and 2. Transferrin sialo-isoforms are indicated by their charge (2-6). B. Normal sialylation of Apo C-III in all four patients before IVIG (Pre), after IVIG loading (Mid), and after the treatment period (Post). Normal control sera $\left(N_{1}\right.$ and $\left.N_{2}\right)$ exhibit three bands; treatment with sialidase (S) reduces this to one main band and a minor band. Samples of patients 3 and 4 were electrophoresed on a gel different from the gel for samples 1 and 2. Apo C-III $I_{0}=$ asialo-; Apo C-III = monosialo-; Apo C-III ${ }_{2}=$ disialo-Apo C-III isoforms.

Click here for file

[http://www.biomedcentral.com/content/supplementary/14712377-7-3-S1.ppt]

\section{Acknowledgements}

This study was supported by the Intramural Research Program of the National Human Genome Research Institute, National Institutes of Health. The authors appreciate the excellent medical care provided by Kevin O'Brien, RN, NP.

\section{References}

I. Griggs RC, Askanas V, DiMaur S, Engel A, Karpati G, Mendell JR, Rowland LP: Inclusion body myositis and myopathies. Ann Neurol 1995, 38:705-7|5.

2. Sadeh M, Gadoth N, Hadar H, Ben-David E: Vacuolar myopathy sparing the quadriceps. Brain 1993, 1 16:217-232.

3. Sivakuma K, Dalakas MC: The spectrum of familial inclusion body myopathies in 13 families and description of a quadriceps sparing phenotype in non Iranian Jews. Neurology 1996, 47:977-984.

4. Argov Z, Eisenberg I, Grabov-Nardini G, Sadeh M, Wirguin I, Soffer $D$, Mitrani-Rosenbaum S: Hereditary inclusion body myopathy. The Middle Eastern genetic cluster. Neurology 2003, 60:1519-1523.

5. Eisenberg I, Avidan N, Potikha T, Hochner H, Chen M, Olender T, Barash M, Shemesh M, Sadeh M, Grabov-Nardini G, Shmilevich I, Friedmann A, Karpati , Bradley WG, Baumbach L, Lancet D, Asher EB, Beckmann JS, Argov Z, Mitrani-Rosenbaum S: The UDP-Nacetylglucosamine 2-epimerase/ $\mathbf{N}$-acetylmannosamine kinase gene is mutated in hereditary inclusion body myopathy. Nat Genet 200I, 29:83-89.

6. Hinderlich S, Stasche R, Zeitler R, Reutter W: A bifunctional enzyme catalyzes the first two steps in $\mathbf{N}$-acetylneuraminic acid biosynthesis of rat liver. Purification and characterization of UDP-N-acetylglucosamine 2-epimerase/ $\mathbf{N}$-acetylmannosamine kinase. J Biol Chem 1997, 272:243 I3-243 I8.

7. Keppler OT, Hinderlich S, Langner J, Schwartz-Albiez R, Reutter W: UDP-GICNAc 2-epimerase: a regulator of cell surface sialylation. Science 1999, 284:1372-1376.

8. Darvish D, Vahedifar P, Huo Y: Four novel mutations associated with autosomal recessive inclusion body myopathy (MIM: 600737). Mol Genet Metab 2002, 77:252-256.

9. Vasconcelos OM, Raghavan R, Dalakas C: GNE mutations in an American family with quadriceps-sparing IBM and lack of mutations in s-IBM. Neurology 2002, 59:1776-I779.

10. Del Bo R, Baron P, Prelle A, Serafini M, Moggio M, Fonzo AD, Castagni $M$, Bresolin N, Comi GP: Novel missense mutation and large deletion of GNE gene in autosomal-recessive inclusionbody myopathy. Muscle Nerve 2003, 28: II 3-II7.

II. Eisenberg I, Grabov-Nardini G, Hochner H, Korner M, Sadeh M, Bertorini T, Bushby K, Castellan C, Felice K, Mendell J, Merlini L, Shilling C, Wirguin I, Argov Z, Mitrani-Rosenbaum S: Mutations spectrum of GNE in hereditary inclusion body myopathy sparing the quadriceps. Hum Mutat 2003, $21: 99$.

12. Broccolini A, Ricci E, Cassandrini D, Gliubizzi C, Bruno C, Tonoli E, Silvestri G, Pescatori M, Rodolico C, Sinicropi S, Servidei S, Zara F, Minetti C, Tonali PA, Mirabella M: Novel GNE mutations in Italian families with autosomal recessive hereditary inclusionbody myopathy. Hum Mutat 2004, 23:632.

13. Kayashima T, Matsuo H, Satoh A, Ohta T, Yoshiura K, Matsumoto N, Nakane $Y$, Niikawa N, Kishino T: Nonaka myopathy is caused by mutations in the UDP- $\mathbf{N}$-acetylglucosamine 2-epimerase/ $\mathbf{N}$ acetylmannosamine kinase gene (GNE). J Hum Genet 2002, 47:77-79.

14. Nishino I, Noguchi S, Murayama K, Driss A, Sugie K, Oya Y, Nagata T, Chida K, Takahashi T, Takusa Y, Ohi T, Nishimiya J, Sunohara N, Ciafaloni E, Kawai M, Aoki M, Nonaka I: Distal myopathy with rimmed vacuoles is allelic to hereditary inclusion body myopathy. Neurology 2002, 59:1689-1693.

15. Tomimitsu H, Ishikawa K, Shimizu J, Ohkoshi N, Kanazawa I, Mizusawa H: Distal myopathy with rimmed vacuoles: Novel mutations in the GNE gene. Neurology 2002, 59:45।-454.

16. Sparks SE, Ciccone C, Lalor M, Orvisky E, Klootwijk R, Savelkoul PJ, Dalakas MC, Krasnewich DM, Gahl WA, Huizing M: Use of a cellfree system to determine UDP-N-acetylglucosamine 2-epimerase and $\mathbf{N}$-acetylmannosamine kinase activities in human Hereditary Inclusion Body Myopathy. Glycobiology 2005, 15:1102-1110. 
17. Noguchi S, Keira Y, Murayama K, Ogawa M, Fujita M, Kawahara G, Oya Y, Imazawa M, Goto Y, Hayashi YK, Nonaka I, Nishino I: Reduction of UDP-N-acetylglucosamine 2-epimerase/ $\mathbf{N}$-acetylmannosamine kinase activity and sialylation in distal myopathy with rimmed vacuoles. I Biol Chem 2004, 279: I I 402-I I 407.

18. Varki A: Diversity in the sialic acids. Glycobiology 1992, 2:25-40.

19. Varki A: Sialic acids as ligands in recognition phenomena. FASEB J 1997, I I:248-255

20. Kelm S, Schauer R: Sialic acids in molecular and cellular interactions. Int Rev Cytol 1997, 175:137-240.

21. Saito F, Tomimitsu H, Aria K, Nakai S, Kanda T, Shimizu T, Mizusawa $\mathrm{H}$, Matsumura $\mathrm{K}$ : A Japanese patient with distal myopathy with rimmed vacuoles: missense mutations in the epimerase domaine of the UDP-N-acetylglucosamine 2-epimerase/ $\mathbf{N}$ acetylmannosamine kinase (GNE) gene accompanied by hyposialiylation of skeletal muscle glycoproteins. Neuromusc Disord 2004, I 4: 158-16I.

22. Tajima Y, Uyama E, Go S, Sato C, Tao N, Kotani M, Hino H, Suzuki A, Sanai Y, Kitajima K, Sakuraba H: Distal Myopathy with Rimmed Vacuoles: Impaired O-glycan formation in muscular glycoproteins. Am J Pathol 2005, 166:II2I-II30.

23. Ricci E, Broccolini A, Gidaro T, Morosetti R, Gliubizzi C, Frusciante $\mathrm{R}$, Di Lella GM, Tonali PA, Mirabella M: NCAM is hyposialylated in hereditary inclusion body myopathy due to GNE mutations. Neurology 2006, 66:755-758.

24. Huizing M, Rakocevic G, Sparks SE, Mamali I, Shatunov A, Goldfarb L, Krasnewich D, Gahl WA, Dalakas MC: Hypoglycosylation of alpha-dystroglycan in patients with hereditary IBM due to GNE mutations. Mol Genet Metab 2004, 81:196-202.

25. Michele DE, Campbell KP: Dystrophin-glycoprotein complex: post-translational processing and dystroglycan function. J Biol Chem 2003, 278: I5457-I5460.

26. Martin PT: Dystroglycan glycosylation and its role in matrix binding in skeletal muscle. Glycobiology 2003, 13:55R-66R.

27. Grewal PK, Hewitt JE: Glycosylation defects: a new mechanism for muscular dystrophy? Hum Mol Genet 2003, I2(Spec No 2):R259-R264

28. Winder SJ: The complexities of dystroglycan. Trends Biochem Sci 2001, 26: II8-124

29. Endo T: Aberrant glycosylation of alpha-dystroglycan and congenital muscular dystrophies. Acta Myol 2005, 24:64-69.

30. Sydow O: Sialic acid content in serum IgG from patients with myotonic dystrophy compared to healthy controls. Acta Neurol Scand 1989, 80:476-478.

3I. Andres PL, Hedlund W, Finison L, Conlon T, Felmus M, Munsat TL: Quantitative motor assessment in amyotrophic lateral sclerosis. Neurology 1986, 36:937-941.

32. Robbins J, Levine R, Wood J, Roecker EB, Luschei E: Age effects on lingual pressure generation as a risk factor for dysphagia. Gerontol A Biol Sci Med Sci 1995, 50:M257-262.

33. Robbins J, Gangnon RE, Theis SM, Kays SA, Hewitt AL, Hind JA: The effects of lingual exercise on swallowing in older adults. J $\mathrm{Am}$ Geriatr Soc 2005, 53: I483-I489.

34. Lin M-R, Hwang H-F, Hu M-H, Wu H-DI, Wang Y-W, Huang F-C: Psychometric comparisons of the timed up and go, one-leg stand, functional reach, and tinetti balance measures in community-dwelling older people. J Am Geriatr Soc 2004, 52:1343-1348

35. ATS statement: Guidelines for the six-minute walk test. Am J Respir Crit Care Med 2002, 166: III-117.

36. van Eijk HG, van Noort WL: The analysis of human serum transferrins with the PhastSystem: quantitation of microheterogeneity. Electrophoresis 1992, 13:354-358.

37. Savelkoul PJM, Manoli I, Sparks S, Ciccone C, Gahl WA, Krasnewich DM, Huizing M: Normal sialylation status of $\mathbf{N}$-linked and $\mathbf{O}$. GalNAc linked glycans in Hereditary Inclusion Body Myopathy. Mol Genet Metab 2006, 88:389-390.

38. Wopereis S, Grunewald S, Monrova E, Penzien JM, Briones P, GarciaSilva MT, Demacker PN, Huijben KM, Wevers RA: Apolipoprotein $\mathrm{C}$-III isofocusing in the diagnosis of genetic defects in O-glycan biosynthesis. Clin Chem 2003, 49:1839-1845.

39. Ronn LC, Berezin $\mathrm{V}$, Bock E: The neural cell adhesion molecule in synaptic plasticity and ageing. Int J Dev Neurosci 2000, 18:193-199.
40. Illa I, Leon-Monzon M, Dalakas MC: Regenerating and denervated human muscle fibers and satellite cells express neural cell adhesion molecule recognized by monoclonal antibodies to natural killer cells. Ann Neurol 1992, 31 :46-52.

4I. Franz CK, Rutishauser U, Rafuse VF: Polysialylated neural cell adhesion molecule is necessary for selective targeting of regenerating motor neurons. J Neurosci 2005, 25:208 I-209|.

42. Covault J, Sanes JR: Neural Cell Adhesion Molecule (N-CAM) accumulates in denervated and paralyzed skeletal muscles. Proc Natl Acad Sci USA 1985, 82:4544-4548.

43. Hinderlich S, Salama I, Eisenberg I, Potikha T, Mantey LR, Yarema KJ, Horstkorte R, Argov Z, Sadeh M, Reutter W, Mitrani-Rosenbaum S: The homozygous M7I2T mutation of UDP-N-acetylglucosamine 2-epimerase/ $\mathbf{N}$-acetylmannosamine kinase results in reduced enzyme activities but not in altered overall cellular sialylation in hereditary inclusion body myopathy. FEBS Lett 2004, 566: 105-109.

44. Salama I, Hinderlich S, Shlomai Z, Eisenberg I, Krause S, Yarema K, Argov Z, Lochmuller H, Reutter W, Dabby R, Sadeh M, Ben-Bassat H, Mitrani-Rosenbaum S: No overall hyposialylation in hereditary inclusion body myopathy myoblasts carrying the homozygous M7I2T GNE mutation. Biochem Biophy Res Comm 2005, 328:22I-226.

45. Broccolini A, Gliubizzi C, Pavoni E, Gidaro T, Morosetti R, Sciandra F, Giardina B, Tonali P, Ricci E, Brancaccio A, Mirabella M: $\alpha$-Dystroglycan does not play a major pathogenic role in autosomal recessive hereditary inclusion-body myopathy. Neuromusc Disord 2005, 15:177-184.

46. Seppala R, Renlund M, Bernardini I, Tietze F, Gahl WA: Renal handling of free sialic acid in normal humans and patients with Salla disease, renal Fanconi syndrome, or glomerular insufficiency. Lab Invest 1990, 63:197-203.

47. Kaneko Y, Nimmerjahn F, Ravetch JV: Anti-inflammatory activity of immunoglobulin $\mathbf{G}$ resulting from Fc sialylation. Science 2006, 3 I 3:670-673

48. Dalakas MC: Intravenous immunoglobulin I auoimmune neuromuscular diseases. JAMA 2004, 291:2367-2375.

49. Aitkens S, Lord J, Bernauer E, Fowler WM, Lieberman JS, Berck P: Relationship of manual muscle testing to objective strength measurements. Muscle Nerve 1989, I 2:173-177.

50. Hinderlich S, Nohring S, Weise C, Franke P, Stasche R, Reutter W: Purification and characterization of $\mathbf{N}$-acetylglucosamine kinase from rat liver-comparison with UDP-N-acetylglucosamine 2-epimerase/ $\mathbf{N}$-acetylmannosamine kinase. Eur J Biochem 1998, 252:133-139.

5I. Schwarzkopf M, Knobelock KP, Rohde E, Hinderlich S, Wiechens N, Lucka L, Horak I, Reutter W, Horstkorte R: Sialylation is essential for early development in mice. Proc Natl Acad Sci USA 2002, 99:5267-5270.

52. Huizing M, Kloowijk R, Galeano B, Manoli I, Sun MS, Ciccone C, Darvish D, Krasnewich D, Gahl WA: N-Acetylmannosamine treatment rescues a mouse model of Hereditary Inclusion Body Myopathy. Glycobiology 2006, I6:I I I0. (Abstr. 35)

\section{Pre-publication history}

The pre-publication history for this paper can be accessed here:

http://www.biomedcentral.com/1471-2377/7/3/prepub 